\title{
Sleep clinical record: an aid to rapid and accurate diagnosis of paediatric sleep disordered breathing
}

\author{
Maria Pia Villa*, Maria Chiara Paolino*, Rosa Castaldo*, Nicola Vanacore", \\ Alessandra Rizzoli*, Silvia Miano*, Marco Del Pozzo* and Marilisa Montesano*
}

ABSTRACT: Overnight polysomnography (PSG) is an expensive procedure which can only be used in a minority of cases, although it remains the gold standard for the diagnosis of sleep disordered breathing (SDB). The objective of this study was to develop a simple, PSG-validated tool to screen SDB, thus reducing the use of PSG.

For every participant we performed PSG and a sleep clinical record was completed. The sleep clinical record consists of three items: physical examination, subjective symptoms and clinical history. The clinical history analyses behavioural and cognitive problems. All three items were used to create a sleep clinical score (SCS).

We studied 279 children, mean \pm SD age $6.1 \pm 3.1$ years, $63.8 \%$ male; $27.2 \%$ with primary snoring and $72.8 \%$ with obstructive sleep apnoea (OSA) syndrome. The SCS was higher in the OSA syndrome group compared to the primary snoring group $(8.1 \pm 9.6$ versus $0.4 \pm 0.3, p<0.005)$, correlated with apnoea/hypopnoea index $(p=0.001)$ and had a sensitivity of $96.05 \%$. Positive and negative likelihood ratios were 2.91 and 0.06 , respectively.

SCS may effectively be used to screen patients as candidates for PSG study for suspected OSA syndrome, and to enable those with a mild form of SDB to receive early treatment.

KEYWORDS: Diagnostic procedures, paediatric sleep disordered breathing, questionnaire

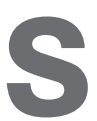
leep disordered breathing (SDB), particularly obstructive sleep apnoea (OSA), is a highly relevant pathology in the paediatric population [1]. $\sim 1 \%$ of children between 5 and 12 years of age were found to have an apnoea/ hypopnoea index $(\mathrm{AHI})>5$ episodes $\cdot \mathrm{h}^{-1}$ [2]. In a recent meta-analysis, the prevalence of habitual snoring as determined by parental report was found to be $7.45 \%$, while the prevalence of OSA ranges from 1 to $4 \%$ [3].

Recently the American Academy of Sleep Medicine (AASM) published practice parameters for polysomnographic indications in children with SDB [4]. The authors reported that the assessment for SDB mostly based on clinical evaluation alone does not have sufficient sensitivity or specificity to establish a diagnosis of OSA [4].

Although overnight polysomnography (PSG) remains the gold standard for the diagnosis of SDB, this procedure is expensive, and in many countries (including Italy) it can only be used in a minority of cases, due to the low number of paediatric sleep centres available, delaying the diagnosis and treatment of patients with SDB.

Several questionnaires have been developed to create a simple screening instrument to identify subjects at high risk for OSA. A systematic review of the literature [5] showed that a patient's clinical history and physical examination are less reliable than PSG to diagnose OSA. CAROLL et al. [6] performed a retrospective study of clinical histories and they did not find clinical differences between primary snoring children and those with OSA, except for mouth breathing. On the contrary, $\mathrm{XU}_{\mathrm{U}}$ et al. [7] demonstrated the potential usefulness of parental observation, the physician's evaluation and radiological findings combined to identify subjects with clinically significant OSA. In 1984, BROUILETTE et al. [8] described a questionnaire

Earn CME accreditation by answering questions about this manuscript. You will find these at the back of the printed copy of this issue or online at www.erj.ersjournals.com/misc/cmeinfo.xhtml

This article has supplementary material available from www.erj.ersjournals.com

AFFILIATIONS

*Paediatric Dept, Faculty of Medicine, "La Sapienza" University, Rome, and

"National Centre for Epidemiology, Surveillance, and Health Promotion, National Institute of Health, Rome, Italy.

CORRESPONDENCE

M.P. Villa

Paediatric Clinic

Sant'Andrea Hospital

Via Grottarossa 1035/1039

00189 Rome

Italy

E-mail: mariapia.villa@uniroma1.it

Received:

Dec 082011

Accepted after revision:

Sept 042012

First published online:

Sept 272012 
designed to detect OSA in children with adenotonsillar hypertrophy on the basis of a prediction score that combined only three nocturnal symptoms (snoring, observed apnoeas and difficulty breathing) yet yielded a high level of accuracy. Li et al. [9] developed the Hong Kong children sleep questionnaire, a questionnaire-based scale composed of items on demography, sleep environment, sleep habits and specific sleep problems; they found that snoring, nocturnal mouth breathing and sweating are highly significant as predictors of the presence of OSA, although the data shed light on single symptoms but do not take into consideration the predictive value of a total score. CHERVIN et al. [10] validated a paediatric sleep questionnaire (PSQ) for sleep disordered breathing, snoring, sleepiness and behavioural problems in 162 children aged 2-18 years. The PSQ was able to predict OSA-related neurobehavioural morbidity and its response to adenotonsillectomy as well as, if not better than, PSG [11]. The main limitation of these questionnaires is that they mostly consider the subject's history and physical examination separately, and that they have rarely been validated for PSG purposes. On the contrary, one study aimed to examine the accuracy of the clinical diagnosis of paediatric OSA by a standardised history, physical examination and review of a tape recording of breathing during sleep of a small sample of 30 children. The authors found that a standardised clinical assessment of OSA in children was sensitive $(92.3 \%)$ but not specific $(29.4 \%)$ for the diagnosis [12].

The aim of this study was to develop a simple, large-sample PSG-validated tool designed 1) to consider both the subject's clinical history and physical examination in the diagnosis of SDB and 2) to be used to screen subjects candidates for a PSGbased diagnosis.

\section{MATERIALS AND METHODS}

We consecutively enrolled children with suspected SDB undergoing a diagnostic assessment for the first time in our Paediatric Sleep Centre (Rome, Italy). A diagnosis of OSA was confirmed by laboratory PSG revealing an obstructive AHI $>1$ event $\cdot \mathrm{h}^{-1}$, according to AASM criteria [13]. A diagnosis of primary snoring was made in children with habitual snoring and an AHI $<1$ event $\cdot h^{-1}$. Patients with a history of epilepsy or of previous treatment for OSA, acute or chronic cardiorespiratory or neuromuscular diseases, dysmorphism, major craniofacial abnormalities or associated chromosomal syndromes were excluded.

A detailed personal and family history was obtained for all the participants and a general clinical examination was performed.

\section{Scale development}

For the purposes of our study, we propose the sleep clinical record (SCR) (see online supplementary material), which combines the patient's history and clinical items. Items were considered to be predictive of SDB in children on the basis of physicians' clinical experience.

The SCR consist of three items. The first point takes into consideration the data yielded by physical examination of the nose, oropharynx and dental and skeletal occlusion, and it reveals the presence of the following: signs of oral breathing, such as nasal cartilage hypotonia, orbicular muscle hypotonia or rhinolalia; nasal obstruction, considering nostril patency and habitual nose obstruction; septum nose deviation; severe tonsillar hypertrophy; dental/skeletal malocclusion; pathologic palate position; narrow palate; and obese or adenoid phenotype.

Tonsillar hypertrophy was graded according to a standardised scale ranging from 0 to 4 . Tonsillar size was graded as: 1+: medial borders of tonsils lateral to or extending to the pillars; 2+: medial borders of tonsils lateral to or extending to the lateral uvular margins; $3+$ : medial borders of tonsils medial to the lateral uvular margins; and 4+: includes "kissing" tonsils, which meet at the midline [14]. We considered grades 3 and 4 as positive.

All the children underwent an orthodontic examination to detect possible jaw deviation from normal occlusion, i.e. deep bite, retrusive bite and cross-bite, or the presence of narrow palate. The palate position was graded according to Friedman classes, with classes 3 and 4 being considered as positive (see online supplementary material) [15]. Nostril patency was analysed by blocking one nostril with one finger, inspiration through the unoccluded nostril, and repetition of the same manoeuvre with the other nostril. The manoeuvre was repeated three times.

The percentile of body mass index (BMI) was also recorded.

The second item investigates the patients' subjective symptoms and their clinical history based on the Brouilette questionnaire [8]. The participants' parents completed a questionnaire on the symptoms of SDB. Questions investigated sleep symptoms (mouth breathing, habitual snoring, witnessed apnoeic episodes, frequent awakenings or agitated sleep) and diurnal symptoms (daytime somnolence and cephalalgia). Questions were kept simple and concise, and a yes/no response format was chosen. We calculated the Brouilette score, considering a score equal to or higher than -1 as positive.

The third item investigates the presence of symptoms of inattention and hyperactivity using the attention deficit hyperactive disorder (ADHD) rating scale [16], adapted to the Italian population [17], which the parents of all the participants were required to fill out. This scale consists of 18 items, divided into two subgroups of nine questions, that investigate symptoms of inattention and hyperactivity. We are aware that this scale is designed for school-aged children, but we did not aim to diagnose ADHD, but to investigate symptoms related to OSA in our population of children.

All these items were used in a model that combines subjective and objective parameters to yield a sleep clinical score (SCS) with the highest sensitivity and negative predictive value. To create this score, we tried several different models in which the points assigned to each item varied. We analysed the sensitivity and specificity of each score in predicting OSA syndrome, defined by PSG, ultimately choosing the one with the best combination of sensitivity, specificity and accuracy. Of the seven different scores we obtained (see online supplementary material), the one with the highest positive predictive value used to define the SCS was yielded by the following formula: $2 \times$ oral breathing $+2 \times$ nasal obstruction $+2 \times$ septum nose deviation $+2 \times$ tonsillar hypertrophy $+2 \times$ Friedman palate position (III-IV) $+2 \times$ dental/skeletal malocclusion $+2 \times$ narrow palate $+2 \times$ phenotype $+0.5 \times$ Brouilette score $+0.5 \times$ other neurological 
symptoms (at least one item) $+1 \times$ hyperactivity/attention rating scale (total score $>6$ ). We performed receiver operating characteristics curve analysis in order to select the optimal one among seven different sleep clinical records. In particular, we estimated the sensitivity, specificity, positive likelihood ratios, negative likelihood ratios and accuracy of each of the seven different SCR clinical scores in predicting OSA. We chose the SCR clinical score with better overall area under the curve (AUC) and sensitivity and specificity than the other six clinical scores.

\section{Sleep validation}

Compilation of the SCR took approximately $30 \mathrm{~min}$ and the clinicians who administered the SCR were blinded to the following PSG-based diagnosis.

The cut-off of the SCR clinical score was identified with the best combination of sensitivity and specificity. Likelihood ratios were also used to calculate post-test probabilities of having OSA based on the SCR clinical score (positive, negative or inconclusive). The split-sample validation test was used to validate SCR clinical score in our single sample.

\section{Polysomnography and sleep stage scoring}

Standard overnight PSG recordings were obtained with a Grass Heritage polygraph (Natus Neurology Incorporated - Grass products, Warwich, RI, USA) on the second night of hospitalisation, after the clinical assessment was completed. The variables recorded included at least a six-channel electroencephalogram, an electro-oculogram, a submental electromyogram and an electrocardiogram. Sleep stages were scored according to the standard criteria of the AASM [13].

Central, obstructive and mixed apnoea events were counted according to the criteria established by the AASM [13]. Chest and abdomen movements were measured by strain gauges. Oronasal airflow was recorded with a thermocouple and nasal pressure was recorded by a nasal cannula. Arterial oxygen saturation was monitored with a pulse oximeter; AHI was defined as the average number of apnoeas and hypopnoeas per hour of sleep. The scorer (S. Miano) was blinded to the SCR results.

The children underwent PSG in our sleep centre after one night of adaptation. The local ethics committee approved the study protocol and all children's parents gave their informed consent to the procedures.

\section{Statistical analysis}

Values are expressed as mean \pm SD. Statistical analysis was performed using the Chi-squared test for categorical variables and t-test for continuous variables. We used Pearson's coefficient to investigate any correlations between the variables studied.

Two-sided p-values were calculated for all the analyses; values of $\leqslant 0.05$ were considered to indicate statistical significance. All statistical analyses were performed with the statistical package SPSS (version 17.0; SPSS Inc., Chicago, IL, USA).

\section{RESULTS}

\section{Study population}

We studied 279 children, mean age $6.1 \pm 3.1$ years, $178(63.8 \%)$ male. The mean AHI was $6.02 \pm 8.8$ events $\cdot \mathrm{h}^{-1}$ (range 060.8 events $\left.\cdot h^{-1}\right), 76(27.2 \%)$ out of 279 children met the criteria for primary snoring (group 0), while 203 (72.8\%) out of 289 children met the criteria for OSA syndrome (group 1).

The children's clinical and anthropometric parameters are shown in table 1, while their PSG respiratory parameters and SCR scores are shown in table 2.

There were no differences for age, sex and percentile of BMI between the two groups. Children with OSA exhibited septum nose deviation, nasal obstruction, signs of mouth breathing and pathological Friedman and tonsillar hypertrophy more frequently than primary snorers.

\section{TABLE 1 Clinical and anthropometric parameters}

\begin{tabular}{|c|c|c|c|}
\hline Subjects & $76(27.2)$ & 203 (72.8) & \\
\hline Centile body mass index $\mathrm{kg} \cdot \mathrm{m}^{-2}$ & $67.4 \pm 34.9$ & $67.1 \pm 37.3$ & NS \\
\hline Male & $54(71)$ & $124(61.1)$ & NS \\
\hline Oral breathing & $24(31.5)$ & $106(52.2)$ & $<0.05$ \\
\hline Tonsillar hypertrophy & $11(14.5)$ & $115(56.6)$ & $<0.001$ \\
\hline Dental/skeletal malocclusion & 58 (76.3) & $171(84.2)$ & NS \\
\hline Friedman palate position III-IV & $10(13.1)$ & $63(31.0)$ & $<0.05$ \\
\hline Narrow palate & $49(64.5)$ & $173(85.2)$ & $<0.001$ \\
\hline Adenoid phenotype & $7(9.2)$ & $38(18.7)$ & NS \\
\hline
\end{tabular}

Data are presented as $\mathrm{n}(\%)$ or mean $\pm \mathrm{SD}$, unless otherwise stated. NS: not significant. ${ }^{\#}$ : limb movements, electroencephalogram paroxysmal activity, daytime somnolence, cephalalgia, enuresis and nocturnal choking. ": p-value calculated by t-test; others calculated by Chi-squared test. 


\begin{tabular}{|c|c|c|c|}
\hline & Primary snoring & Obstructive sleep apnoea & p-value \\
\hline \multicolumn{4}{|l|}{ Respiratory parameters } \\
\hline AHI events $\cdot h^{-1}$ & $0.4 \pm 0.3$ & $8.1 \pm 9.6$ & $<0.001$ \\
\hline Oxygen saturation \% & $97.6 \pm 1.2$ & $96.7 \pm 1.9$ & $<0.001$ \\
\hline SCS & $6.3 \pm 2.9$ & $9.4 \pm 2.5$ & $<0.001$ \\
\hline \multicolumn{4}{|c|}{ Other alternative scores } \\
\hline Score 1 & $4.06 \pm 1.8$ & $5.4 \pm 1.5$ & $<0.001$ \\
\hline Score 2 & $5.2 \pm 2.4$ & $7.1 \pm 2.2$ & $<0.001$ \\
\hline Score 3 & $6.0 \pm 2.5$ & $6.8 \pm 2.1$ & $<0.001$ \\
\hline Score 4 & $4.4 \pm 2.4$ & $5.6 \pm 2.2$ & $<0.001$ \\
\hline
\end{tabular}

Data are presented as $n(\%)$ or mean \pm SD, unless otherwise stated. p-values calculated by t-test. AHI: apnoea/hypopnoea index; SCS: sleep clinical score; NS: not significant.

\section{Sleep clinical score}

The SCS showed an AUC (mean \pm SE) of $0.804 \pm 0.35$ (CI 95\% 0.736-0.872; $\mathrm{p}=0.001$ ) (fig. 1).

The SCS was defined as positive when it was $\geqslant 6.25$. We investigated correlations between the various scores and the severity of OSA as expressed by the AHI and the mean arterial oxygen saturation $\left(\mathrm{SaO}_{2}\right)$. The SCS showed a positive correlation with AHI $(\mathrm{p}<0.001)$ (table 3$)$. No correlation emerged between the scores and the mean $\mathrm{SaO}_{2}$.

The SCS was positive in 220 out of the 279 children studied; the PSG yielded a diagnosis of OSA in 195 of these 220 children (true positives (TP)) and of primary snoring in the remaining 25 children (false positives (FP)). The clinical characteristics of the two groups revealed that FP children had other neurological symptoms (limb movements, electroencephalogram (EEG) paroxysmal activity, daytime somnolence, cephalalgia, enuresis or nocturnal choking) more frequently than TP children (14 (56\%) out of 25 versus $44(22 \%)$ out of $195, p=0.001)$.

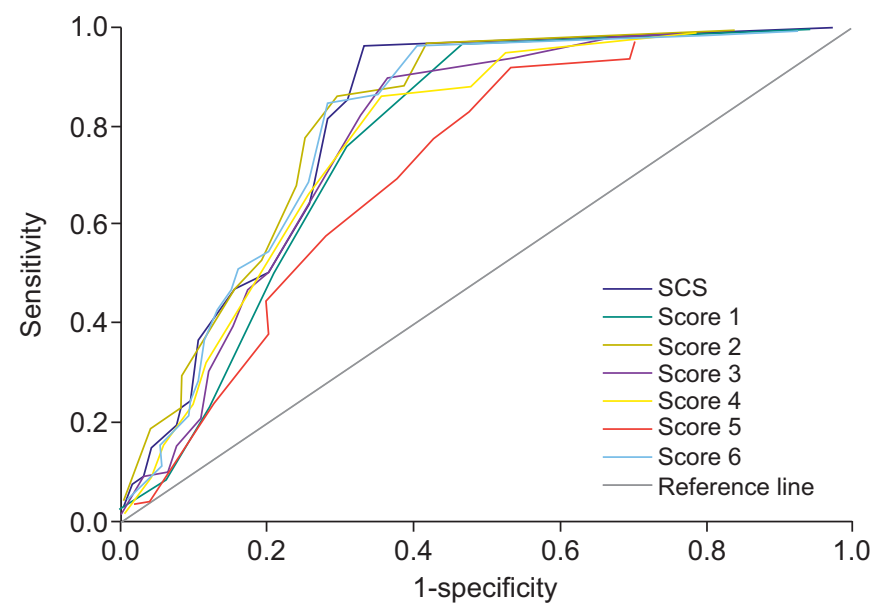

FIGURE 1. Receiver operating characteristics curve analysis. SCS: sleep clinical score.
59 children had a negative SCS; the PSG diagnosed OSA (false negative (FN)) in eight of these children. True negative children were older than FN children (mean age $6.8 \pm 3.0$ years versus $4.6 \pm 1.8$ years, $\mathrm{p}<0.05)$. Of the 203 children with a PSG diagnosis of OSA (AHI >1), 195 were TP, while eight children had a negative SCS (FN). Between these two groups there were no differences for age, sex and BMI centile. FN children had a significantly lower AHI than TP children $\left(2.6 \pm 1.3\right.$ events $\cdot h^{-1}$ (range 1.2-5) versus $8.2 \pm 9.7$ events $\cdot h^{-1}$ (range 1.1-60.8), $\mathrm{p}<0.001$ ). FN children had nasal obstruction and severe tonsillar hypertrophy less frequently than TP children (nasal obstruction: four (50\%) out of eight versus 175 (89.7\%) out of 195, $\mathrm{p}<0.01$; severe tonsillar hypertrophy: one (14.3\%) out of seven versus 115 $(58.9 \%)$ out of $195, \mathrm{p}<0.05)$.

The SCS had a sensitivity of $96.05 \%$, a specificity of $67 \%$, positive likelihood ratio of 2.91, negative likelihood ratio of 0.06 and accuracy of $88.2 \%$.

A positive SCS $(\geqslant 6.5)$ had a likelihood ratio of 2.91, which increased the probability of having OSA to $89 \%$. A negative or inconclusive SCS $(<6.5)$ had a likelihood ratio of 0.06 , which lowered the probability of having OSA of $14 \%$.

The results of the split-sample validation test showed that $89.2 \%$ of the subjects had been classified correctly.

Finally, we subdivided the sample into two groups: children who had tonsillar hypertrophy (grade III and IV) and children who did not. Table 4 shows the statistical differences between the two groups. Children with tonsillar hypertrophy showed a higher SCS and AHI, and a lower oxygen saturation and body mass index compared to those without tonsillar hypertrophy.

\section{DISCUSSION}

To our knowledge, the SCS is the first instrument validated on a large sample of children for the diagnosis of OSA, based on a combination of physical examination of the patient and case history. Our results demonstrate that the SCS has a high sensitivity and a high negative likelihood ratio, identifying children with primary snoring or a mild form of OSA. 
TABLE 3 Correlations between clinical scores and polysomnographic respiratory parameters

\begin{tabular}{|c|c|c|c|c|}
\hline & \multicolumn{2}{|c|}{ Apnoea hypopnoea index events $\cdot h^{-1}$} & \multicolumn{2}{|c|}{ Mean oxygen saturation \% } \\
\hline & Pearson's correlation & $\mathrm{p}$-value & Pearson's correlation & p-value \\
\hline Sleep clinical score & 0.216 & $0.001^{\star \star}$ & -0.096 & 0.102 \\
\hline \multicolumn{5}{|c|}{ Other alternative scores } \\
\hline Score 1 & 0.092 & 0.119 & -0.077 & 0.189 \\
\hline Score 4 & 0.145 & $0.013^{*}$ & -0.080 & 0.177 \\
\hline Score 5 & -0.004 & 0.942 & -0.035 & 0.559 \\
\hline Score 6 & 0.144 & $0.014^{\star}$ & -0.109 & 0.064 \\
\hline
\end{tabular}

This means that SCS may accurately exclude the diagnosis with the result that no further testing, and of course no treatment, is necessary.

In contrast, several questionnaires have been validated to identify subjects at high risk for OSA [5, 7,8]. Our results support the idea that the combination of clinical examination and patient history has a strong predictive value, at least to detect children at low risk of developing a severe form of OSA. A previous study aimed to examine the accuracy of the clinical diagnosis of paediatric OSA, evaluating 30 children with obstructive symptoms by means of a standardised history, physical examination and review of a tape recording of breathing during sleep [12]. The authors found a high sensitivity but a low specificity to this assessment, and this result is probably due to the low numbers of children assessed [12].

Although PSG remains the gold standard for the diagnosis of $\mathrm{SDB}$, this expensive procedure is usually used in a minority of cases owing to the limited availability of paediatric sleep centres. As early treatment of OSA is necessary to prevent its neurocognitive, behavioural, cardiovascular and metabolic consequences, early diagnosis of the disorder is critical. The main consequence of repetitive apnoeas and hypopnoeas during sleep is intermittent hypoxia, which is a potent trigger of oxidative stress and inflammation [18-22]. Other mechanisms through which OSA may cause complications include increased sympathetic activity [23, 24], increased serum cortisol [25], hypoxia-induced hormonal

TABLE 4 Statistical differences between children with tonsillar hypertrophy compared to those without

\begin{tabular}{|c|c|c|c|}
\hline Subjects $n$ & $126(45.2)$ & $153(54.8)$ & \\
\hline Centile body mass index $\mathrm{kg} \cdot \mathrm{m}^{-2}$ & $59.4 \pm 36.9$ & $76.6 \pm 35.1$ & $<0.001^{\circ}$ \\
\hline Apnoea/hypopnea index events $\cdot h^{-1}$ & $8.9 \pm 10.5$ & $3.5 \pm 6.3$ & $<0.001^{\circ}$ \\
\hline Mean oxygen saturation \% & $96.7 \pm 2.1$ & $97.2 \pm 1.3$ & $<0.01^{\bullet}$ \\
\hline Oral breathing & $63(50)$ & $67(43.7)$ & $\mathrm{NS}^{+}$ \\
\hline Nasal obstruction & $110(87.3)$ & $120(78.4)$ & $\mathrm{NS}^{+}$ \\
\hline Septum nose deviation & $12(9.5)$ & $19(12.4)$ & $\mathrm{NS}^{+}$ \\
\hline Dental/skeletal malocclusion & $103(81.7)$ & $126(82.3)$ & $\mathrm{NS}^{+}$ \\
\hline Friedman palate position III-IV & $40(31.7)$ & $33(21.5)$ & $\mathrm{NS}^{+}$ \\
\hline Rating scales positive for hyperactivity or attention deficit & $23(18.2)$ & $41(26.8)$ & $\mathrm{NS}^{+}$ \\
\hline Other neurological symptoms ${ }^{\#}$ & $18(14.2)$ & $60(39.2)$ & $<0.001^{+}$ \\
\hline
\end{tabular}

Data are presented as $\mathrm{n}(\%)$ or mean $\pm \mathrm{SD}$, unless otherwise stated. *: limb movements, electroencephalogram paroxysmal activity, daytime somnolence, cephalalgia, enuresis or nocturnal choking; NS: not significant. ${ }^{\circ}:$ p-values calculated by t-test; ${ }^{+}$: p-values calculated by Chi-squared test. 
changes, arousal from sleep and secondary sleep debt [26-28]. Although audio-video taping and overnight pulse-oximetry may be used as first-line investigations, their quality varies considerably and they require strong parental motivation $[29,30]$. Overnight oximetry only identifies the subset of patients with more severe OSA, and even this goal is not fully achieved because marked oxyhaemoglobin desaturation is not present in all patients with severe OSA [29]. The information ruled out by the SCS can be useful to identify children with SDB who can wait, deferring PSG recording, and can directly receive an early diagnosis and treatment. This is also supported by the fact that the few subjects having a false negative score showed a lower $\mathrm{AHI}$, and a lower percentage of nasal obstruction and of severe tonsillar hypertrophy than children with a true positive score. Since they expressed a mild form of OSA they also can forego a PSG and be directly treated. According to our findings, the SCS may obviate the need for a PSG study in approximately 21\% (59 out of 279) of our sample, thus reducing waiting lists and costs.

Conversely, a positive correlation between the SCS and AHI was found in our sample, and the SCS would also be useful to screen patients for the presumably severe form, necessitating an urgent recording. The relatively low specificity of the SCS is due to the high number of false positives found. The group of children with a false positive score showed a higher frequency of other neurological symptoms (limb movements, EEG paroxysmal activity, daytime somnolence, cephalalgia, enuresis or nocturnal choking) compared with those with a true positive score. In our opinion, the presence of neurological symptoms in children with positive SCS is in any case an additional reason to perform a nocturnal PSG recording in order to exclude the presence of other sleep-related neurological disorders, mimicking sleep obstructive disordered breathing (such as sleep central apnoeas, nocturnal epilepsy or periodic limbs movements) [31]. An indirect confirmation of these results is the higher SCS found in children with tonsillar hypetrophy compared with those without. Children with tonsillar hypertrophy also had a higher AHI, confirming that tonsillar hypertrophy in association with other risk factors, such as dental malocclusions, adenoid hypertrophy, narrow palate and/ or obesity increase the risk of a more severe form of OSA.

In our study, we did not find any differences in the percentage of children with a positive ADHD rating scale score between subjects with OSA and primary snorers. It is extensively reported that children with SDB display diurnal neurobehavioural problems such as ADHD, learning problems, behavioural disorders and hypersomnolence [32,33]. Although a significant improvement in diurnal neurobehavioral disorders has been widely demonstrated following adenotonsillectomy [32, 34-36], the relationship between the severity of OSA syndrome and cognitive deficits is usually weak [37-39]. The results of our study support the idea these symptoms are related to SDB but are not specific to OSA.

One limitation of the sleep record we propose is that it was validated in a selected population of children with suspected SDB in whom OSA accounted for $\sim 72 \%$ of the cases, and it has not been validated in normal children. This tool should thus be used by experts in sleep disorders or paediatricians on children who are referred to physicians for snoring, apnoea or oral breathing during sleep, not as a screening instrument in the general paediatric population.
In addition, this instrument may effectively be used to identify patients with OSA, and the specificity and the positive predictive value may be increased by adding other screening instruments such as nocturnal pulse-oximetry.

\section{Conclusions}

SCS is an instrument that may effectively be used to screen patient candidates for PSG study for suspected OSA, and those with a mild form of SDB, enabling early treatment.

\section{SUPPORT STATEMENT}

This study was supported by Capgemini Italia S.p.A.

\section{STATEMENT OF INTEREST}

None declared.

\section{REFERENCES}

1 Rosen CL, Larkin EK, Kirchner HL, et al. Prevalence and risk factors for sleep-disordered breathing in 8- to 11-year-old children: association with race and prematurity. J Pediatr 2003; 142: 383-389.

2 Bixler EO, Vgontzas AN, Lin HM, et al. Sleep disordered breathing in children in a general population sample: prevalence and risk factors. Sleep 2009; 32: 731-736.

3 Lumeng JC, Chervin RD. Epidemiology of pediatric obstructive sleep apnea. Proc Am Thorac Soc 2008; 5: 242-252.

4 Aurora RN, Zak RS, Karippot A, et al. Practice parameters for the respiratory indications for polysomnography in children. Sleep 2011; 34: 379-388.

5 Brietzke SE, Katz ES, Roberson DW. Can history and physical examination reliably diagnose paediatric obstructive sleep apnea/ hypopnea syndrome? A systemic review of the literature. Otolaryngol Head Neck Surg 2004; 131: 827-832.

6 Carroll JL, McColley SA, Marcus CL, et al. Inability of clinical history to distinguish primary snoring from obstructive sleep apnea syndrome in children. Chest 1995; 108: 610-618.

$7 \mathrm{Xu} \mathrm{Z}$, Cheuk DK, Lee SL. Clinical evaluation in predicting childhood obstructive sleep apnea. Chest 2006; 130: 1765-1771.

8 Brouilette R, Hanson D, David R, et al. A diagnostic approach to suspected obstructive sleep apnea in children. J Pediatr 1984; 105: 10-14.

9 Li AM, Cheung A, Chan D, et al. Validation of a questionnaire instrument for prediction of obstructive sleep apnea in Hong Kong Chinese children. Pediatr Pulmonol 2006; 41: 1153-1160.

10 Chervin RD, Hedger K, Dillon JE, et al. Pediatric sleep questionnaire (PSQ): validity and reliability of scales for sleep-disordered breathing, snoring, sleepiness, and behavioural problems. Sleep Med 2000; 1: 21-32.

11 Chervin RD, Weatherly RA, Garetz SL, et al. Pediatric sleep questionnaire: prediction of sleep apnea and outcomes. Arch Otolaryngol Head Neck Surg 2007; 133: 216-222.

12 Goldstein NA, Sculerati N, Walsleben JA, et al. Clinical diagnosis of pediatric obstructive sleep apnea validated by polysomnography. Otolaryngol Head Neck Surg 1994; 111: 611-617.

13 Iber C, Ancoli-Israel S, Chesson AL, et al. The AASM Manual for the Scoring of Sleep and Associated Events: Rules, Terminology, and Technical Specifications. 1st Edn. Westchester, American Academy of Sleep Medicine, 2007.

14 Liistro G, Rombaux P, Belge C, et al. High Mallampati score and nasal obstruction are associated risk factors for obstructive sleep apnoea. Eur Respir J 2003; 21: 248-252.

15 Friedman M, Ibrahim H, Joseph NJ. Staging of obstructive sleep apnea/hypopnea syndrome: a guide to appropriate treatment. Laryngoscope 2004; 114: 454-459.

16 DuPaul GJ, McGoey KE, Eckert TL, et al. Preschool children with attention-deficit/ hyperactivity disorder: impairments in behavioral, 
social, and school functioning. J Am Acad Child Adolesc Psychiatry 2001; 40: 508-515.

17 Marzocchi GM, Cornoldi C. Una scala di facile uso per la rilevazione dei comportamenti problematici dei bambini con Deficit di Attenzione e Iperattività. [A scale to indentify behavioural problems in ADHD children.] Psicologia Clinica dello Sviluppo 2000; 43-64.

18 Lavie L. Obstructive sleep apnoea syndrome - an oxidative stress disorder. Sleep Med Rev 2003; 7: 35-51.

19 Larkin EK, Rosen CL, Kirchner HL, et al. Variation of C-reactive protein levels in adolescents: association with sleep disordered breathing and sleep duration. Circulation 2005; 111: 1978-1984.

20 Tauman R, O'Brien LM, Gozal D. Hypoxemia and obesity modulate plasma C-reactive protein and interleukin-6 levels in sleep-disordered breathing. Sleep Breath 2007; 11: 77-84.

21 Kaditis A, Gozal D, Snow AB, et al. Uric acid excretion in North American and Southeast European children with obstructive sleep apnea. Sleep Med 2010; 11: 489-493.

22 Verhulst SL, Van Hoeck K, Schrauwen N, et al. Sleep-disordered breathing and uric acid in overweight and obese children and adolescents. Chest 2007; 132: 76-80.

23 Aljadeff G, Gozal D, Schechtman VL, et al. Heart rate variability in children with obstructive sleep apnea. Sleep 1997; 20: 151-157.

24 Kaditis AG, Alexopoulos EI, Damani E, et al. Urine levels of catecholamines in Greek children with obstructive sleep-disordered breathing. Pediatr Pulmonol 2009; 44: 38-45.

25 Bratel T, Wennlund A, Carlström K. Pituitary reactivity, androgens and catecholamines in obstructive sleep apnoea. Effects of continuous positive airway pressure treatment (CPAP). Respir Med 1999; 93: 1-7.

26 Spiegel K, Knutson K, Leproult R, et al. Sleep loss: a novel risk factor for insulin resistance and type 2 diabetes. J Appl Physiol 2005; 99: 2008-2019.

27 Flint J, Kothare SV, Zihlif M, et al. Association between inadequate sleep and insulin resistance in obese children. J Pediatr 2007; 150: 364-369.

28 Verhulst SL, Schrauwen N, Haentjens D, et al. Sleep duration and metabolic dysregulation in overweight children and adolescents. Arch Dis Child 2008; 93: 89-90.
29 Section on Pediatric Pulmonology, Subcommittee on Obstructive Sleep Apnea Syndrome, American Academy of Pediatrics. Clinical practice guidelines: diagnosis and management of childhood obstructive sleep apnea syndrome. Pediatrics 2002; 109: 704-712.

30 Collop NA, Anderson WM, Beoehlecke B, et al. Clinical guidelines for the use of unattended portable monitors in the diagnosis of obstructive sleep apnea in adult patients. Portable Monitoring Task Force of the American Academy of Sleep Medicine. J Clin Sleep Med 2007; 3: 737-747.

31 Miano S, Bachiller C, Gutiérrez M, et al. Paroxysmal activity and seizures associated with sleep breathing disorder in children: a possible overlap between diurnal and nocturnal symptoms. Seizure 2010; 19: 547-552.

32 Gozal D. Obstructive sleep apnea in children: implications for the developing central nervous system. Semin Pediatr Neurol 2008; 15 : 100-106.

33 Chervin RD, Ruzicka DL, Archbold KH, et al. Snoring predicts hyperactivity four years later. Sleep 2005; 28: 885-890.

34 Owens-Stively J, Frank N, Smith A, et al. Child temperament, parenting discipline style, and daytime behavior in childhood sleep disorders. J Dev Behav Pediatr 1997; 18: 314-321.

35 Montgomery-Downs HE, Crabtree VM, Gozal D. Cognition, sleep and respiration in at-risk children treated for obstructive sleep apnoea. Eur Respir J 2005; 25: 336-342.

36 Mitchell RB, Kelly J. Long-term changes in behavior after adenotonsillectomy for obstructive sleep apnea syndrome in children. Otolaryngol Head Neck Surg 2006; 134: 374-378.

37 Décary A, Rouleau I, Montplaisir J. Cognitive deficits associated with sleep apnea syndrome: a proposed neuropsychological test battery. Sleep 2000; 23: 369-381.

38 Friedman BC, Hendeles-Amitai A, Kozminsky E, et al. Adenotonsillectomy improves neurocognitive function in children with obstructive sleep apnea syndrome. Sleep 2003; 26: 999-1005.

39 Miano S, Paolino MC, Urbano A, et al. Neurocognitive assessment and sleep analysis in children with sleep-disordered breathing. Clin Neurophysiol 2011; 122: 311-319. 